\title{
Freqüência de Síndrome Metabólica em Pacientes com Nefrite Lúpica
}

\section{Frequency of Metabolic Syndrome in Patients with Lupus Nephritis}

\author{
Bernardo Matos da Cunha ${ }^{(1)}$, Evandro Mendes Klumb ${ }^{(2)}$, Jaqueline Quintanilha Moura ${ }^{(3)}$, Amanda Santos ${ }^{(3)}$, \\ Geraldo da Rocha Castelar Pinheiro ${ }^{(4)}$, Elisa M. N. Albuquerque ${ }^{(5)}$, Roger Abramino Levy(4)
}

\section{RESUMO}

Introdução: Os pacientes com lúpus eritematoso sistêmico (LES) apresentam morbimortalidade importante por doenças cardiovasculares (DCV). A síndrome metabólica (SM) é um transtorno complexo representado por um conjunto de fatores de risco para DCV. Objetivos: Avaliar a prevalência de SM em uma coorte de pacientes com nefrite lúpica em seguimento em um hospital universitário brasileiro, e o seu impacto na doença, além da freqüência de cada fator de risco cardiovascular analisado individualmente. Métodos: Quarenta e seis pacientes com nefrite lúpica foram estudados e classificados de acordo o critério para SM da National Cholesterol Education Program - Adult Treatment Panel III (NCEP-ATP III). Resultados: A SM esteve presente em 30,4\% dos pacientes. Foi observada correlação linear entre a presença de SM e níveis baixos de depuração de creatinina e valores altos de creatinina sérica ( $\mathrm{p}<0,04$ e p $<0,008$, respectivamente). Não houve correlação com a classificação de nefrite lúpica, duração da doença, dose cumulativa de prednisona, proteinúria de 24 horas e com o escore Systemic Lupus International Collaborating Clinics (SLICC). Conclusão: Os pacientes brasileiros com nefrite lúpica estudados apresentam alta prevalência de $S M$, comparável a de estatísticas internacionais. Os únicos fatores relacionados à SM nessa população foram os que definem a presença de dano renal crônico.

Palavras-chave: lúpus, nefrite, síndrome metabólica.

\section{INTRODUÇÃO}

A mortalidade de pacientes com lúpus eritematoso sistêmico (LES) é maior que a da população geral pareada por idade e gênero e está estreitamente vinculada às lesões causadas pela doença, bem como à terapia imunossupres-

\begin{abstract}
Introduction: patients with systemic lupus erythematosus (SLE) have $n$ increased morbidity due to cardiovascular diseases (CVD). Metabolic syndrome (MS) is a complex syndrome composed by a cluster of cardiovascular risk factors. Objectives: study the prevalence of MS in a cohort of Brazilian patients with lupus nephritis in a university hospital; evaluate its impact in disease outcome, and the frequency of each specific risk factor. Methods: 46 patients with lupus nephritis were studied and defined as having or not MS in accordance with the National Cholesterol Education Program - Adult Treatment Panel III (NCEP-ATP III) criteria. Results: MS was present in $30.4 \%$ of patients. There was significant association between MS presence and low levels of creatinine clearance and high levels of blood creatinine $(p<0.04$ and $p<0.008$, respectively). The class of lupus nephritis, the disease duration, cumulative dose of prednisone, 24-hour urine protein and Systemic Lupus International Collaborating Clinics (SLICC) score were not significantly associated with MS. Conclusion: In our cohort of patients with lupus nephritis there was a high prevalence of MS, comparable to international data. The only factors associated with MS in that population were the ones that define chronic renal damage.
\end{abstract}

Keywords: lupus, nephritis, metabolic syndrome.

sora empregada nos casos mais graves ${ }^{(1)}$. Até a década de 1950, todos os estudos sobre o assunto apontavam como principal causa de morte, nesses pacientes, o acometimento renal e do sistema nervoso central, seguido das infecções. Nessa época, a taxa de mortalidade entre pacientes com LES era de $50 \%$ em três anos ${ }^{(1)}$. A partir do início do uso

\footnotetext{
Recebido em 21/12/2007. Aprovado, após revisão, em 26/6/2008. Agência financiadora: Programa Institucional de Bolsas de Iniciação Científica (PIBIC)/UERJ. Disciplina de Reumatologia da Faculdade de Ciências Médicas da Universidade do Estado do Rio de Janeiro (UERJ).

1. Reumatologista (título SBR), médico do serviço de clínica médica do Hospital da Lagoa, ex-residente do serviço de Reumatologia do Hospital Universitário Pedro Ernesto da Universidade do Estado do Rio de Janeiro (Hupe-UERJ).

2. Reumatologista, médico do serviço de Reumatologia do Hupe-UERJ.

3. Estudante de medicina, estagiária do serviço de Reumatologia do Hupe-UERJ

4. Professor adjunto do serviço de reumatologia do Hupe-UERJ.

5. Professora assistente do serviço de Reumatologia do Hupe-UERJ.

Endereço para correspondência: Roger A. Levy, Hupe-UERJ - Reumatologia, Boulevard 28 de setembro, 77, 3ªndar, sala 333, 20551-900, Rio de Janeiro, RJ, e-mail: roger.a.levy@gmail.com
} 
de corticosteróides no tratamento do LES em 1956, do emprego de diuréticos sulfonamídicos e da disponibilidade de procedimentos de diálise em 1963, houve progressiva redução da mortalidade que, em 1966, já era de 30\% em cinco $\operatorname{anos}^{(2)} \mathrm{e}, \mathrm{em}$ 1976, 7\% em cinco anos ${ }^{(3)}$.

Com a melhora dos meios de diagnóstico, do tratamento da doença e das medidas de suporte à vida, houve aumento progressivo da sobrevida e, em 1976, Urowitz et al. ${ }^{(2)}$ observaram padrão bimodal de causas de óbito, sendo o primeiro pico relacionado à atividade de doença e infecções e o segundo às doenças arteriais coronarianas (DAC). Nos anos subseqüentes, outros autores também observaram o mesmo padrão que ficou conhecido como a curva bimodal de mortalidade de Urowitz. Estudos realizados comparando a mortalidade por DAC em pacientes com LES e na população geral evidenciaram número significativamente maior nos primeiros, principalmente entre as mulheres na faixa etária de 35 a 44 anos, com risco relativo variando de 2,67 e $50^{(4-8)}$. Quando comparados à população geral, os pacientes com LES apresentam maior número de fatores de risco clássicos para DAC, com menores níveis de colesterolHDL e maiores da fração LDL, triglicerídeos, lipoproteína A, creatinina e homocisteína. Também foi encontrada maior freqüência de pacientes com diabetes melito concomitante, hiperinsulinemia, resistência insulínica, hipertensão arterial sistêmica e maiores índices da relação cintura/quadril( ${ }^{(9-12)}$. Como conseqüência, quando pareados para gênero e idade com indivíduos da população geral, os portadores de LES apresentam aterosclerose acelerada, conforme observado pela presença de placas de ateroma em carótidas, defeitos de perfusão na cintilografia miocárdica e alteração do índice tornozelo-braquial ${ }^{(13-18)}$.

A síndrome metabólica (SM) é um transtorno complexo representado por um conjunto de fatores de risco para doenças cardiovasculares, que inclui principalmente a resistência à insulina, a dislipidemia e a deposição central de tecido adiposo. A SM contribui, por sua vez, para aumento da mortalidade geral em 1,5 vez e a para doença cardiovascular em 2,5 vezes ${ }^{(19,20)}$. No Brasil, a SM constitui problema de saúde pública, pois, não obstante sua prevalência ser menor do que em países da Europa e dos Estados Unidos, é distúrbio freqüente na população brasileira, acometendo entre $7,5 \%$ e $30 \%$ da população geral ${ }^{(21,22)}$.

Recentemente Chung et al. ${ }^{(23)}$, em estudo realizado na Holanda, demonstraram maior freqüência da SM em pacientes com $\mathrm{LES}^{(23)}$, o que foi confirmado por outro grupo da Holanda, com incidência de $16 \%^{(24)}$, e também por pesquisadores brasileiros, com incidência variando de $20 \%$ a $28,4 \%{ }^{(25,26)}$.

O objetivo deste estudo foi avaliar a freqüência de $S M$ em pacientes brasileiros com nefrite lúpica atendidos em um ambulatório de um hospital universitário. Como objetivos secundários, foram analisados a correlação entre a presença de SM e os dados da doença de base e a freqüência de cada fator de risco cardiovascular individualmente, incluindo aqueles que não compunham a definição de SM.

\section{PACIENTES E MÉTODOS}

Foram recrutados, seqüencialmente, por meio de corte transversal, pacientes do ambulatório de lúpus da Disciplina de Reumatologia da Faculdade de Ciências Médicas da Universidade do Estado do Rio de Janeiro (FCM-UERJ), no período entre março e agosto de 2007.

Foram incluídos no estudo pacientes com LES segundo os critérios do Colégio Americano de Reumatologia revisados em $1997^{(27)}$ e nefrite lúpica, com idade igual ou maior que 18 anos e tendo ou não, como comorbidades, outras doenças reumáticas auto-imunes ou doenças cardiovasculares. Foram classificados portadores de nefrite lúpica os pacientes que apresentassem alterações histológicas renais compatíveis com o diagnóstico de nefrite lúpica classes III, IV ou V, de acordo com a classificação da International Society of Nephrology/Renal Pathology Society, de $2003^{28}$ ou da Organização Mundial da Saúde (OMS), conforme originalmente descrito à época da biópsia, ou proteinúria inicial > 1 g/24 horas, sendo excluídos dessa classificação os pacientes que apresentassem histologia renal compatível com nefropatia associada à síndrome antifosfolipídio isolada.

Foram excluídos os pacientes que não apresentavam nefrite em sua história clínica, doença renal crônica terminal, transplantados ou em diálise e os com doença renal não associada diretamente ao LES (inclusive nefropatia diabética). Os pacientes foram avaliados a partir de entrevista padronizada, avaliação clínica, bem como revisão de prontuário e solicitação de exames complementares, no dia de sua consulta. As informações referentes às condições socioeconômicas foram obtidas segundo critérios definidos pela Associação Brasileira de Empresas de Pesquisa (ABEP). Os dados antropométricos (peso corporal, altura, índice de massa corpórea (IMC), circunferência abdominal e relação cintura/quadril), pressões arterial (PA) sistólica e diastólica aferida nos membros superiores, freqüência cardíaca, assim como dados da doença de base, medicações em uso ou já utilizadas, hábitos de vida incluindo tabagismo, etilismo 
e atividade física e histórias clínicas pessoal e familiar de doença cardiovascular foram obtidos no dia da avaliação. No dia da consulta, foram coletadas amostras de sangue periférico, após jejum de 12 horas, para avaliação do colesterol total e frações, triglicerídeos, glicemia, creatinina sérica, relação proteinúria/creatininúria em amostra e depuração da creatinina, calculada a partir da fórmula de Cockroft-Gault; hábitos de vida incluindo tabagismo, etilismo e atividade física e história clínica pessoal e familiar de doença cardiovascular.

Os dados referentes à doença de base incluíram: o tempo de doença, a classificação de nefrite lúpica, conforme os critérios descritos anteriormente, o estágio de doença renal crônica (DRC), definido segundo a classificação da Kidney Disease Outcomes Quality Initiative (KDOQI) ${ }^{(29)}$, as doses cumulativas de prednisona e a avaliação do índice de danos permanentes por meio da escala do Systemic Lupus International Collaborating Clinics (SLICC) ${ }^{(30)}$. Para a classificação da SM nos pacientes com LES, foi utilizada a definição adotada pela I Diretriz Brasileira de Diagnóstico e Tratamento da Síndrome Metabólica ${ }^{(31)}$, a qual se fundamenta na definição do National Cholesterol Education Program-Adult Treatment Panel III (NCEP-ATP III) ${ }^{(32)}$. Segundo essa classificação, a SM é caracterizada pela presença de, pelo menos, três dos seguintes critérios: circunferência abdominal $>102 \mathrm{~cm}$ em homens e $>88 \mathrm{~cm} \mathrm{em}$ mulheres; triglicerídeos $\geq 150 \mathrm{mg} / \mathrm{dL}$; colesterol-HDL $<40 \mathrm{mg} / \mathrm{dL}$ em homens e $<50 \mathrm{mg} / \mathrm{dL}$ em mulheres; pressão arterial sistólica $\geq 130 \mathrm{mmHg}$ ou pressão arterial diastólica $\geq 85 \mathrm{mmHg}$; e glicemia de jejum $\geq 110 \mathrm{mg} / \mathrm{dL}$. A estratificação dos níveis pressóricos foi definida conforme a classificação do Seventh Report of the Joint National Committee on Prevention, Detection, Evaluation, and Treatment of High Blood Pressure ${ }^{(33)}$. O colesterol-LDL ótimo foi definido de acordo com a classificação do NCEP-ATP III. A classificação do IMC foi realizada a partir da definição adotada pela $\mathrm{OMS}^{(34)}$.

Foi realizada análise exploratória dos dados, comparando as características da doença em pacientes com e sem SM com a utilização de testes paramétricos, como o teste exato de Fisher ou teste $t$ de Welch para variáveis categóricas. As variáveis numéricas foram comparadas por meio do teste de Wilcoxon. Todas as análises utilizaram nível de significância estatística de $5 \%$ e foram realizados com o programa $\mathrm{R}$ versão 2.6.1 (www.r-project.org).

O estudo foi aprovado pelo comitê de ética em pesquisa do hospital e todos os participantes assinaram termo de consentimento livre e esclarecido.

\section{RESULTADOS}

\section{CARACTERÍSTICAS GERAIS DOS PACIENTES}

Foram incluídos 42 pacientes do sexo feminino e quatro do sexo masculino, com idade média de 40,8 (mediana 40 \pm 11 ) anos. Os dados de etnia, grau de escolaridade e dados socioeconômicos não foram diferentes entre os pacientes com e sem SM.

\section{DADOS DA DOENÇA}

Os pacientes apresentavam tempo médio de doença de 147,77 meses ( 14 a 321,50 ). Trinta e quatro pacientes $(73 \%)$ haviam sido submetidos à biópsia renal, sendo 11 (23,90\%) classificados como classe III, $13(28,20 \%)$ como classe IV, 11 $(23,90 \%)$ como classe V e um (1) como glomeruloesclerose segmentar e focal (GESF). Alguns pacientes têm mais de uma classificação. O SLICC médio do grupo foi de 1,2 (0-4). Em 41 pacientes, foi possível calcular a dose cumulativa de prednisona e seu valor médio foi de $31,70 \mathrm{~g}(5,10$ a 95,60). $\mathrm{Na}$ avaliação da função renal, a creatinina média foi de $1,12 \mathrm{mg} / \mathrm{dL}(0,45$ a 3,37$)$, a depuração média de creatinina de $90,87 \mathrm{ml} / \mathrm{min}(20$ a 197) e a proteinúria média de $0,99 \mathrm{~g}$ $(0,01$ a 4,18$)$ (esta última calculada entre 32 pacientes). A média, a mediana e o desvio-padrão destes dados se encontram na Tabela 1 . Foram classificados $23(50 \%)$ pacientes como portadores de DRC estágio I, dez $(21,70 \%)$ como estágio II, nove $(19,50 \%)$ como estágio III, quatro $(8,70 \%)$ como estágio IV e nenhum paciente como estágio $\mathrm{V}$.

\section{SÍNDROME METABÓLICA}

Os critérios para o diagnóstico de SM foram preenchidos por 30,4\% dos pacientes da amostra. Entre os homens, apenas um caso foi definido como portador da síndrome. Não foi encontrada correlação entre as características gerais dos pacientes e a presença de SM. Quando separados pela classificação histológica da nefrite, foi encontrada SM entre seis $(54,54 \%)$ dos pacientes com lesão classe III, dois $(15,38 \%)$ dos pacientes com lesão classe IV e dois dos pacientes com lesão classe $V$, não havendo diferenças na freqüência de $S M$ entre essas classes $(p>0,2)$. Foi observada correlação com níveis baixos de depuração de creatinina $(\mathrm{p}<0,04)$ e com valores altos de creatinina sérica ( $\mathrm{p}<0,008)$, mas não com a proteinúria de 24 horas. Não foram encontradas diferenças significantes estatisticamente em relação ao tempo médio de doença, escore do SLICC e dose cumulativa média de corticóides. Estes dados se encontram detalhados na Tabela 1. 
Tabela 1

Correlação entre os dados da doença e a SM

\begin{tabular}{|c|c|c|c|c|c|c|}
\hline Parâmetro & Média total & Mediana & DP & SM (+) (média) & SM (-) (média) & $\mathrm{p}$ \\
\hline Duração da doença (meses) & 142,8 & 120,8 & $\pm 87,75$ & 134,5 & 146,5 & 0,504 \\
\hline Dose cumulativa de prednisona $(\mathrm{g})$ & 31,7 & 23,9 & $\pm 23,2$ & 37,61 & 29,3 & 0,656 \\
\hline Creatinina (mg/dL) & 1,12 & 0,89 & $\pm 0,61$ & 1,51 & 0,94 & 0,007 \\
\hline Depuração da creatinina (ml/min) & 90,87 & 87 & $\pm 43,34$ & 69,15 & 99,19 & 0,033 \\
\hline Proteinúria de 24 horas (g) & 0,99 & 0,45 & $\pm 1,18$ & 1,1 & 0,94 & 0,76 \\
\hline SLICC & $\mathbf{n}$ & SM (+) & SM (-) & $\mathrm{p}$ & & \\
\hline 0 & 18 & 4 & 14 & & & \\
\hline 1 & 9 & 3 & 6 & & & \\
\hline 2 & 12 & 5 & 7 & 0,864 & & \\
\hline 3 & 4 & 1 & 3 & & & \\
\hline 4 & 3 & 1 & 2 & & & \\
\hline Classificação nefrite & $\mathbf{n}$ & SM (+) & SM (-) & $\mathrm{p}$ & & \\
\hline III & 11 & 6 & 5 & & & \\
\hline IV & 13 & 2 & 11 & & & \\
\hline V & 11 & 2 & 9 & 0,206 & & \\
\hline GESF & 1 & 1 & 0 & & & \\
\hline Sem biópsia & 13 & 4 & 9 & & & \\
\hline
\end{tabular}

DP = desvio-padrão; SM (+) = presença de SM; SM $(-)$ = ausência de SM.

\section{FATORES DE RISCO CARDIOVASCULARES}

A média, a mediana e o desvio-padrão dos valores dos dados clínicos e laboratoriais se encontram na Tabela 2 e sua estratificação em categorias com valores em porcentagens na Tabela 3. Como se pode observar, os pacientes deste estudo apresentavam, de maneira geral, sobrepeso e hipertrigliceridemia. Em contrapartida, nenhum dos pacientes do estudo apresentava hiperglicemia.

TABEla 2

MÉdia, MEdiana E DESVIO-PAdRÃo dos FATORES DE RISCO CARDIOVASCULARES

\begin{tabular}{lccc}
\hline Parâmetro & Média & Mediana & DP \\
\hline IMC & 26,44 & 25,96 & 5,72 \\
\hline PA sistólica (mmHg) & 123,98 & 120 & 19,62 \\
\hline PA diastólica (mmHg) & 80,76 & 80 & 14,87 \\
\hline Cintura abdominal & 91,39 & 90 & 11,36 \\
\hline LDL (mg/dL) & 130,33 & 122 & 45,16 \\
\hline HDL (mg/dL) & 52,72 & 50 & 13,79 \\
\hline Triglicerídeos (mg/dL) & 153,54 & 132 & 75,52 \\
\hline Glicose (mg/dL) & 74,30 & 73 & 17,96 \\
\hline
\end{tabular}

Tabela 3

FATORES DE RISCOS CARDIOVASCULARES ESTRATIFICADOS

\begin{tabular}{|c|c|c|}
\hline Parâmetro & $\mathbf{n}$ & $\%$ \\
\hline \multicolumn{3}{|l|}{ IMC } \\
\hline Normal & 20 & 0,44 \\
\hline$\geq 25$ & 26 & 0,56 \\
\hline Total & 46 & 1 \\
\hline \multicolumn{3}{|c|}{ PA sistólica (mmHg) } \\
\hline$<120$ & 15 & 0,33 \\
\hline$\geq 120$ & 31 & 0,67 \\
\hline Total & 46 & 1 \\
\hline \multicolumn{3}{|c|}{ PA diastólica $(\mathrm{mmHg})$} \\
\hline$<80$ & 15 & 0,33 \\
\hline$\geq 80$ & 31 & 0,67 \\
\hline Total & 46 & 1 \\
\hline \multicolumn{3}{|c|}{ Cintura abdominal (cm) (mulheres/homens) } \\
\hline$\leq 88 / 102$ & $20 / 4$ & $0,48 / 1$ \\
\hline$>88 / 102$ & $22 / 0$ & $0,52 / 0$ \\
\hline Total & $42 / 4$ & 1,00 \\
\hline \multicolumn{3}{|c|}{ LDL (mg/dL) } \\
\hline$<100$ & 11 & 0,24 \\
\hline$\geq 100$ & 35 & 0,76 \\
\hline Total & 46 & 1 \\
\hline \multicolumn{3}{|c|}{ HDL (mg/dL) (mulheres/homens) } \\
\hline$<40 / 50$ & $22 / 0$ & $0,48 / 0$ \\
\hline$\geq 40 / 50$ & $20 / 4$ & $0,52 / 1$ \\
\hline Total & $42 / 4$ & $1 / 1$ \\
\hline \multicolumn{3}{|c|}{ Triglicerídeos $(\mathrm{mg} / \mathrm{dL})$} \\
\hline$\leq 150$ & 25 & 0,54 \\
\hline$>150$ & 21 & 0,46 \\
\hline Total & 46 & 1 \\
\hline \multicolumn{3}{|c|}{ Glicose $(\mathrm{mg} / \mathrm{dL})$} \\
\hline$<110$ & 46 & 1 \\
\hline$>110$ & 0 & 0,00 \\
\hline Total & 46 & 1 \\
\hline
\end{tabular}




\section{DISCUSSÃO}

A SM é distúrbio freqüente em diversas populações e parece ter importância semelhante na população brasileira. O Brasil ainda carece de estudos de porte para avaliação da questão $^{(31)}$. O presente estudo verificou alta freqüência de SM nos pacientes com LES e nefrite tratados no Serviço de Reumatologia do Hupe-UERJ. Por conta do desenho deste estudo, embora não seja possível a comparação direta com a população geral, quando é feita a comparação da freqüência de SM nesta população de pacientes, observa-se que esta é maior do que em indivíduos da população geral de outros estados $^{(21)}$ e também maior do que em outra população com LES no nordeste do Brasil ${ }^{(25)}$, embora menor do que a do estudo realizado em Minas Gerais ${ }^{(26)}$.

Os únicos dados que apresentaram correlação com a presença de SM foram os níveis de creatinina sérica e depuração de creatinina, que traduzem o dano renal crônico destes pacientes, mas não a proteinúria, que é, por sua vez, marcador de doença ativa. É possível que o aumento da freqüência de SM e fatores de risco cardiovasculares nos pacientes com LES não esteja relacionado à inflamação sistêmica ou ao dano relacionado à doença, mas seja decorrente da alteração crônica da função renal. Já foi demonstrada a relação entre $S M^{(24)}$, progressão de placas carotídeas ${ }^{(35,36)} \mathrm{e}$ disfunção renal em outras populações com LES e também em populações variadas, por isso, os achados do presente estudo só corroboram o que já se conhecia em relação a outros grupos de pacientes. De acordo com os achados deste estudo, não está claro de que modo estão relacionadas com a gravidade da doença, a intensidade da imunossupressão e a presença de SM.

Os pacientes do trabalho em análise apresentavam, em média, tempo de doença, SLICC e dose cumulativa de corticosteróides bem mais elevados do que no estudo de Chung et al. ${ }^{(23)}$ tendo, portanto, maior morbidade conseqüente à doença e ao tratamento instituído. Ainda assim, a prevalência de SM encontrada no presente estudo foi comparável ao outro, mesmo em se tratando de populações diferentes entre si, o que pode representar a existência de algum fator presente de modo constante nos pacientes com LES, não relacionado com a gravidade da doença e que altera o risco cardiovascular de maneira independente dos fatores de risco clássicos. Essa hipótese foi lançada por Esdaile et al..$^{(37)}$ e confirmada por Lee et al. ${ }^{(38)}$ e Ahmad et al. ${ }^{(39)}$, quando demonstraram, em uma série de pacientes com LES, que as diferenças no perfil de fatores de riscos clássicos entre pacientes com LES e a população geral não eram suficientes para explicar o aumento da incidência de doença aterosclerótica. No estudo de Esdaile et $a l{ }^{\left({ }^{37)}\right.}$, a incidência de IAM não-fatal foi dez vezes maior do que a esperada de acordo com a escala de Framingham, a de morte por DAC foi 17 vezes maior e a de acidente vascular encefálico teve aumento próximo de oito vezes. Com efeito, outros autores têm evidenciado novos fatores diretamente relacionados à inflamação sistêmica e possivelmente implicados no mecanismo de aterogênese acelerada, como a ativação do sistema complemento, inibição da lipoproteína lipase pela IL- $6^{(8)}$, anticorpos anti-HDL e anti-Ox LDL ${ }^{(10)}$, além da presença dos anticorpos antifosfolipídicos, que foram associados à reatividade cruzada com anti-HDL e anti-ApoAl, anti-OxLDL e à formação de complexos $\beta 2$ GP1-OxLDL ${ }^{(11,40,41)}$. Outros trabalhos estabeleceram relações entre doença cardiovascular e manifestações de atividade de doença, como pericardite ${ }^{(41)}$, vasculite digital ${ }^{(6)}$, aumento da VHS e da $\mathrm{PCR}^{(4)}$ e leucopenia ou com escores mais elevados de SLICC ${ }^{(42)}$.

Nesse sentido, o tratamento do LES com medicações imunossupressoras pode ser vantajoso na inibição do processo de aterosclerose. Roman et al. ${ }^{(17)}$ demonstraram menor índice de aterosclerose de carótidas nos pacientes que utilizaram pequenas doses de prednisona e Ahmad et al. ${ }^{(43)}$ demonstraram que os pacientes com LES que utilizaram maiores quantidades de hidroxicloroquina e ciclofosfamida tiveram menor incidência de placas carotídeas.

Uma das manifestações mais graves e importantes do LES é a nefrite, cuja evolução pode levar à DRC, que está associada a risco cardiovascular aumentado. Embora tenhase observado a presença de SM em porcentagens progressivamente maiores, de maneira inversamente proporcional à depuração de creatinina, houve proporção semelhante de pacientes com SM em comparação ao estudo holandês citado anteriormente ${ }^{(23)}$, apesar de este ter sido composto por pacientes com depuração média de creatinina maior e também freqüência de SM quase idêntica à do estudo realizado em Minas Gerais, mesmo em se tratando de população de LES geral ${ }^{(26)}$, mostrando que a DRC pode não ter sido o único fator determinante da presença de SM. Os achados deste estudo têm implicação prática no sentido de alertar os reumatologistas brasileiros quanto à importância que os fatores de risco cardiovasculares podem ter na população de pacientes com LES, estimulando maior atenção sobre as medidas de prevenção a serem instituídas nesses pacientes. 


\section{REFERÊNCIAS}

1. Urman JD, Rothfield NF: Corticosteroid treatment in systemic lupus erythematosus: survival studies. JAMA 238: 2272-6, 1977.

2. Urowitz MB, Bookman AA, Koehler BE, et al.: The bimodal mortality pattern of systemic lupus erythematosus. Am J Med 60: 221-5, 1976.

3. Wallace DJ, Podell T, Weiner J, et al.: Systemic lupus erythematosus survival patterns. Experience with 609 patients. JAMA 245: 934-8, 1981.

4. Karsh J, Klippel JH, Balow JE, et al.: Mortality in lupus nephritis. Arthritis Rheum 22: 764-9, 1979.

5. Pistiner M, Wallace DJ, Nessim S, et al.: Lupus erythematosus in the 1980s: a survey of 570 patients. Semin Arthritis Rheum 21: 55-64, 1991.

6. Manzi S, Meilahn EN, Rairie JE, et al.: Age-specific incidence rates of myocardial infarction and angina in women with systemic lupus erythematosus: comparison with the Framingham Study. Am J Epidemiol 145: 408-15, 1997.

7. Ward MM: Premature morbidity from cardiovascular and cerebrovascular diseases in women with systemic lupus erythematosus. Arthitis Rheum 42: 338-46, 1999.

8. Fischer LM, Schlienger RG, Matter C, et al.: Effect of rheumatoid arthritis or systemic lupus erythematosus on the risk of first-time acute myocardial infarction. Am J Cardiol 93: 198-200, 2004.

9. Bruce IN, Urowitz MB, Gladman DD, et al.: Risk factors for coronary heart disease in women with systemic lupus erythematosus: the Toronto Risk Factor Study. Arthritis Rheum 48: 3159-67, 2003

10. Svenungsson E, Jensen-Urstad K, Heimburger M, et al.: Risk factors for cardiovascular disease in systemic lupus erythematosus. Circulation 104: 1887-93, 2001

11. Bruce IN: 'Not only... but also': factors that contribute to accelerated atherosclerosis and premature coronary heart disease in systemic lupus erythematosus. Rheumatology 44: 1492-502, 2005.

12. El-Magadmi M, Ahmad Y, Turkic W, et al.: Hyperinsulinaemia, insulin resistance and circulating oxidised low density lipoprotein in women with systemic lupus erythematosus. J Rheumatol 33(1): 50-6, 2006.

13. Bruce IN, Burns RJ, Gladman DD, Urowitz MB: Single photon emission computed tomography dual isotope myocardial perfusion imaging in women with systemic lupus erythematosus. I. Prevalence and distribution of abnormalities. J Rheumatol 27: 2372-7, 2000.

14. Sella EM, Sato EI, Leite WA, et al.: Myocardial perfusion scintigraphy and coronary disease risk factors in systemic lupus erythematosus. Ann Rheum Dis 62: 1066-70, 2003.

15. Theodoridou A, Bento L, D'Cruz DP, et al.: Prevalence and associations of an abnormal ankle-brachial index in systemic lupus erythematosus: a pilot study. Ann Rheum Dis 62: 1199 203,2003

16. Hosenpud JD, Montanaro A, Hart MV, et al.: Myocardial perfusion abnormalities in asymptomatic patients with systemic lupus erythematosus. Am J Med 77: 286-92, 1984.

17. Roman MJ, Shanker BA, Davis A, et al.: Prevalence and correlates of accelerated atherosclerosis in systemic lupus erythematosus. N Engl J Med 349: 2399-406, 2003.
18. Souza AWS, Hatta FS, Miranda JR F, et al.: Placas ateroscleróticas em carótidas em pacientes com lúpus eritematoso sistêmico: freqüência e fatores de risco associados. Sao Paulo Med J 123(3): 137-42, 2005

19. Lakka HM, Laaksonen DE, Lakka TA, et al.: The metabolic syndrome and total and cardiovascular disease mortality in middle-aged man. JAMA 288: 2709-16, 2002.

20. Girman CJ, Rhodes T, Mercuri M, et al.: For the $4 \mathrm{~S}$ group and the AFCAPS/TexCAPS research group. The metabolic syndrome and risk of major coronary events in the Scandinavian Sinvastatin Survival Study (4S) and the Air Force/Texas Coronary Atherosclerosis Prevention Study (AFCAPS/TexCAPS). Am J Cardiol 93: 136-41, 2004

21. Barbieri MA, Bettiol H, Silva AAM, et al.: Health in early adulthood: the contribution of the 1978/79 Ribeirão Preto birth cohort. Braz J Med Biol Res 39(8): 1041-55, 2006.

22. Oliveira EP, Souza MLA, Lima MDA: Prevalência de síndrome metabólica em uma área rural do semi-árido baiano. Arq Bras Endocrinol Metab 50(3): 456-65, 2006.

23. Chung CP, Avalos I, Oeser A, et al.: High prevalence of the metabolic syndrome in patients with systemic lupus erythematosus: Association with disease characteristics and cardiovascular risk factors. Ann Rheum Dis 66: 208-14, 2007.

24. Bultink IE, Turkstra F, Diamant M, et al.: Prevalence of and risk factors for the metabolic syndrome in women with systemic lupus erythematosus. Clin Exp Rheumatol 26(1): 32-8, 2008.

25. Azevedo JD, Gadelha RGN, Vilar MJ: Metabolic syndrome in systemic lupus erythematosus: lower prevalence in Brazil than in USA. Ann Rheum Dis 66: 1542, 2007.

26. Telles RW, Lanna CCD, Ferreira GA, et al.: Freqüência de doença cardiovascular aterosclerótica e de seus fatores de risco em pacientes com lúpus eritematoso sistêmico. Rev Bras Reumatol 47(3): 165-73, 2007.

27. Hochberg MC: Updating the American College of Rheumatology revised criteria for the classification of systemic lupus erythematosus. Arthritis Rheum 40: 1725, 1997.

28. Weening JJ, D'Agati VD, Schwartz MM, et al.: The classification of glomerulonephritis in systemic lupus erythematosus revisited. J Am Soc Nephrol 15: 241-50, 2004.

29. K/DOQI. Clinical practice guidelines for chronic kidney disease: evaluation, classification and stratification. Am J Kidney Dis 39:2(Suppl 1): S1-000, 2002.

30. Gladman DD, Urowitz MB, Goldsmith $\mathrm{CH}$, et al.: The reliability of the Systemic Lupus International Collaborating Clinics/ American College of Rheumatology Damage Index in patients with systemic lupus erythematosus. Arthritis Rheum 40(5): 809-13, 1997

31. Brandão AP, Brandão AA, Nogueira AR, et al.: I Diretriz Brasileira de Diagnóstico e Tratamento da Síndrome Metabólica. Rev Bras Hipertens 7(4): 124-59, 2004.

32. Third report of the National Cholesterol Education Program (NCEP) Expert Panel on detection, evaluation, and treatment of high blood cholesterol in adults (Adult Treatment Panel III). Circulation 106: 3143, 2002.

33. Chobanian AV, Bakris GL, Black HR, et al.: The Seventh Report of the Joint National Committee on Prevention, Detection, Evaluation, and Treatment of High Blood Pressure: the JNC 7 report. JAMA 289(19): 2560-72, 2003. 
34. World Health Organization. Obesity: preventing and managing the global epidemic. Report of a WHO convention, Geneva, 1999. WHO technical report series 894, Geneva 2000.

35. Thompson T, Sutton-Tyrrell K, Wildman RP, et al.: Progression of carotid intima-media thickness and plaque in women with systemic lupus erythematosus. Arthritis Rheum 58(3): 835-42, 2008.

36. Maksimowicz-McKinnon K, Magder LS, Petri M: Predictors of carotid atherosclerosis in systemic lupus erythematosus. J Rheumatol 33(12): 2458-63, 2006.

37. Esdaile JM, Abrahamowicz M, Grodzicky T, et al.: Traditional Framingham risk factors fail to fully account for accelerated atherosclerosis in systemic lupus erythematosus. Arthritis Rheum 44(10): 2331-7, 2001.

38. Lee $\mathrm{AB}$, Godfrey $\mathrm{T}$, Rowley $\mathrm{KG}$, et al.: Traditional risk factor assessment does not capture the extent of cardiovascular risk in systemic lupus erythematosus. Intern Med J 36(4): 237-43, 2006.

39. Ahmad Y, Shelmerdine J, Bodill H, et al.: Subclinical atherosclerosis in systemic lupus erythematosus (SLE): the relative contribution of classic risk factors and the lupus phenotype. Rheumatology 46(6): 983-8, 2007.

40. Delgado JA, Kumar S, Isenberg DA: Cross-reactivity between anti-cardiolipin, anti-high-density lipoprotein and antiapolipoprotein A-I IgG antibodies in patients with systemic lupus erythematosus and primary antiphospholipid syndrome. Rheumatology 42: 893-9, 2003.

41. Kobayashi K, Kishi M, Atsumi T, et al.: Circulating oxidized LDL forms complexes with beta2-glycoprotein I: implication as an atherogenic autoantigen. J Lipid Res 44: 716-26, 2003.

42. Selzer F, Sutton-Tyrrell K, Fitzgerald SG, et al.: Comparison of risk factors for vascular disease in the carotid artery and aorta in women with systemic lupus erythematosus. Arthritis Rheum 50: 151-9, 2004.

43. Ahmad Y, Bodill H, Shelmerdine J, et al.: Antiphospholipid antibodies (APLA) contribute to atherogenesis in SLE. Arthritis Rheum 50(Suppl 1): S191, 2004. 